\title{
Correction: Heat Generation by Ion Friction in Water under Alternative Electric Field
}

\author{
[J. Korean Phys. Soc. 75, 832 (2019)]
}

DOI: $10.3938 / j k p s .75 .832$

\author{
Yana Sissembayeva, Jinpho Hong, Hong Yeol Park, Yoon-Hwae Hwang* and Hyung Kook KIM ${ }^{\dagger}$ \\ Department of Nano Fusion Technology, Pusan National University, Busan 46241, Korea \\ Young Tae KIM \\ SJT Co., Ltd, Samjin Hydroheat, Busan 48472, Korea \\ Moonil $\mathrm{KIM}^{\ddagger}$ \\ Department of Environmental Administration, Catholic University of Pusan, Busan 46252, Korea
}

DOI: $10.3938 /$ jkps.75.1054

In author list, the second author's name should be changed from "Jinpho HONG" to "Jinpyo HONG".

\footnotetext{
*E-mail: yhwang@pusan.ac.kr

${ }^{\dagger}$ E-mail: hkkim@pusan.ac.kr

${ }_{\ddagger}$ E-mail: mikim@cup.ac.kr
} 